\title{
Alternating Hemiplegia of Childhood and Neurological Comorbidities. Variable Intrafamilial Clinical Features.
}

Piero Pavone ( $\sim$ ppavone@unict.it)

University of Catania https://orcid.org/0000-0002-5600-9560

\section{Xena Giada Pappalardo}

Universita degli Studi di Catania

\section{Naira Mustafa}

University of Cambridge

\section{Sung Yoon Cho}

Sungkyunkwan University School of Medicine

Dong Kyu Jin

Sungkyunkwan University School of Medicine

\section{Gemma Incorpora}

Azienda Ospedaliero Universitaria Policlinico Vittorio Emanuele Catania

\section{Raffaele Falsaperla}

Azienda Ospedaliero Universitaria Policlinico Vittorio Emanuele Catania

\section{Simona Domenica Marino}

Azienda Ospedaliero Universitaria Policlinico Vittorio Emanuele Catania

\section{Giovanni Corsello}

Universita degli Studi di Palermo

\section{Enrico Parano}

"Consiglio Nazionale delle Ricerche"

Martino Ruggieri

Azienda Ospedaliero Universitaria Policlinico Vittorio Emanuele Catania

\section{Case report}

Keywords: Alternating Hemiplegia of Childhood (AHC), Epilepsy, Developmental Delay, Flunarizine, ATP1A3, GRIN2A, SCN1B, KCNQ2

Posted Date: September 21st, 2020

DOl: https://doi.org/10.21203/rs.3.rs-73126/v1 
License: (c) (i) This work is licensed under a Creative Commons Attribution 4.0 International License. Read Full License 


\section{Abstract}

BACKGROUND Alternating Hemiplegia of Childhood (AHC) is an uncommon and complex disorder characterized by age of onset before 18 months, recurrent hemiplegia of one or either sides of the body or quadriplegia. Developmental delay, epilepsy, tonic or dystonic spells, nystagmus and autonomic manifestations are frequently reported manifestations. AHC is mainly caused by mutations in ATP1A3 gene, and to a lesser extent in ATP1A2 gene.

CASE PRESENTATION Clinical and genetic findings of a couple of twins and a couple of siblings affected by AHC from two different Italian families were deeply examined. Intrafamilial clinical variability was shown in the present cases. A pathogenic variant rs606231437 in ATP1A3 gene was detected in twins, while in one of the siblings was found a novel variant of GRIN2A (c.3175T>A) gene, shared by the other brother who also had a variant in $S C N 1 B(\mathrm{c} .632 \mathrm{G}>\mathrm{A})$ and $K C N Q 2$ (c.1870G>A) genes.

CONCLUSIONS Developmental delay, epileptic seizures and motor dysfunction are features frequently associated to paroxysmal hemiplegic attacks. Hemiplegic episode is only a sign even if the most remarkable of several neurological comorbidities in AHC carriers. Therefore, we suggest that the view of the disorder should be greatly changed to the term "AHC spectrum disorder". The comparison of molecular analysis among the four probands brings out how the genetic framework is not recurrent, but may result from an unexpected greater genetic heterogeneity, such as seen in siblings carrying a new set of gene variants implicated in channelopathies likely to be eligible as further risk factors for AHC.

\section{Highlights}

- Alternating Hemiplegia of Childhood (AHC) has a variable penetrance.

- Defects of ATP1A3 gene are mainly involved in AHC.

- We analyzed a couple of twins and siblings affected by AHC from two families.

- New pathogenic candidates (GRIN2A, SCN1B and KCNQ2) for AHC have been identified.

- A novel genotype-phenotype correlation can be assumed.

\section{Introduction}

Alternating Hemiplegia of Childhood (AHC) is an uncommon complex disorder, characterized mainly by paroxysmal episodes of repeated, transient paresis involving either or both sides of the body with onset usually before the age of 18 months. Subjects with AHC also present with non-paroxysmal features including Developmental Delay/Intellectual Disability (DD/ID), epileptic seizures, autonomic dysfunction, abnormal eye movements, motor impairment, ataxia, dystonia and movement disorders such as choreoathetosis [1-3]. This disorder was first described in late 1960s by Verret and Steele on their study, which included eight children with migraine, among whom three showed the characteristic features of AHC [1]. 
Although the pathophysiologic mechanisms causing the clinical expression of the disorder remain unknown, significant research advances over the years, particularly those seen in the clinical genetics field, allowed for a better mechanistic understanding of the attributed genes and enabled early diagnosis and effective treatment. Two types of AHC have been described; AHC-1 (OMIM\#104290) and AHC-2 (OMIM\#614820), characterized by mutations in ATP1A2 and ATP1A3 genes respectively, which encode two different alpha subunits of the $\mathrm{Na}^{+} / \mathrm{K}^{+}$ATPase transmembrane ion pump $[4,5]$. ATP1A3 is by far more common than ATP1A2 mutation [6]. It is worth mentioning that clinical expression of ATP1A3 mutations are expanding and includes the complex disorders ATP1A3-related [7]. The rise and widespread implementation of the whole exome sequencing (WES) technology has notably enhanced the gene mutation panel analyzing additional genes clinically-overlapped with the AHC spectrum. It is essential to highlight that $\mathrm{AHC}$ as observed in the literature is a complex disorder in which the paroxysmal hemiplegia is only one feature, even if the most relevant, among several other clinical manifestations each of which is considered as a primary component of this disorder. Consequently, we agree that the extensive term "AHC spectrum disorder" may be correctly applied.

Herewith we report on the main neurological manifestations occurring in association to the AHC and specifically DD/ID and seizures, the intrafamilial clinical variability as regards to the frequency and intensity both of the hemiplegic episodes, and the correlated non-paroxysmal manifestations as observed in the cases here reported. We also evaluate the role of a novel gene set detected by WES analysis thought to be likely eligible as risk factors for AHC.

\section{Methods}

This study follows criteria developed by the CARE statement (https://www.care-statement.org/checklist).

\section{Case studies presentation}

\section{Patient Information of Family 1 (Twin pair)}

Twin girls were born to unrelated Italian parents. The clinical features and course of the disorder was rather similar in both girls but one of whom exhibited milder manifestations as regards the frequency and the intensity of the AHC episodes, in both of twins the ID was mild. A wider clinical manifestation of the twins has been previously reported [8]. The clinical onset started in their first few months of life with paroxysmal events bath-induced lasted two years. At the age of 2 years, both twins had an episode of acute encephalopathy rapidly solved. Around 3 years, they presented with typical recurrent episodes of hemiplegia prevailing in the right side. Signs of distonic movements were further noted. In the following years, a marked reduction to disappearance of the hemiplegic attacks were observed with mild persistence of the other disturbances. During the adolescence, in both girls the degree of ID remaimed mild/moderate, instead brief dystonic movements and severe episodes of headache were registered (Table 1). 


\section{Patient Information of Family 2 (Sibling pair)}

The siblings' mother had atresia of the proximal part of the bile ducts and was admitted for liver transplantation in the first months of her life. Both parents are neurologically normal and denied to have suffered from paralysis or epileptic seizures.

\section{a. Sibling 1}

A 10-year-old male patient, first came to the Unit of Pediatrics and Pediatric Emergency Hospital "Policlinico-Vittorio Emanuele", University of Catania. Italy at the age of three months for seizures. He was born at the 39th week of gestation by cesarean section due to the threatened abortion and internal podalic version. The first three months of life passed uneventfully with the exception of two episodes of partial seizures lasting about few minutes and not associated with fever. On admission, trans-fontanellar ultrasound and electroencephalogram (EEG) recordings were normal as well routine laboratory analyses. The child was discharged on Valproate treatment at the dosage of $20 \mathrm{mg} / \mathrm{Kg} / \mathrm{day}$. In the subsequent months two other epileptic episodes were recorded. At 18 months, he showed difficulty in standing up without support. The delay also involved the language development. At this age, the boy manifested an episode of generalized hypertonia of short duration followed by right hemiparalysis resolved after 48 hours initially referred to as Todd's paralysis, but the clinical suspect of alternating hemiplegia of childhood (AHC) came out. At the age of two years, the hemiplegic attacks were frequent as well as tonic and dystonic attacks. The child showed to be apathetic with poor social skills. At the age of two years and nine months, the boy continued to present unfrequent epileptic seizures (1-2 for months) no coincidentally alternated with episodes of hemiparesis mainly right sided. Treatment with flunarizine $10 / \mathrm{mg} /$ day was started. On examination after the hemiparetic episode the affected side of the body was hypotonic, there was difficulty to stimulate patellar reflexes, and reduction of tactile, thermal, and pain sensibility were also noted. Some months later, the clinical manifestations of AHC involved alternatively both sides, and sometimes both sides at the same time. Video-EEG at awake and during the sleep showed multifocal spike and waves mainly expressed in the frontal region. Treatment with valproate was maintained plus levetiracetam as an add-on. During the following years, the epileptic seizures and paretic episodes were frequent and poorly responsive to any type of treatment including flunarizine, anticonvulsants, and ketogenic diet. The hemiplegic manifestations showed a wide variability with some episodes lasting for one hour, and others being prolonged to several days up to 2-3 months. At the age of 10 years old, the child showed a fairly good condition at physical examination. He attended the regular school with sufficient scholastic performance. At neurological examination, pain sensitivity was found to be reduced. Brain Magnetic Resonance Imaging (MRI) was normal. Serial EEG in the awake state and during the sleep showed the presence of multiple spike and waves mainly expressed in the frontal region (a seizure recorded during the EEG registration) (Fig. 1a-b). At the most recent follow-up visit at the Institution (11 years old), the child reported that he attends the school regularly with support. He had a mild ID (IQ = 58) WISC III. He had very few clinical manifestations, and the attacks of hemiparesis and 
seizures became less frequent and less severe compared to the past. He is still taking phenobarbital and lorazepam.

\section{b. Sibling 2}

This is a $6 \frac{1}{2}$ years old male patient. He was born at the 37 th week of gestation by cesarean section. The mother complained that the child was suffering from frequent episodes of upper airways infections and nocturnal cyanotic crises with breathing cessation due to obstructive sleep apnea syndrome (OSAS). The child grew up normally without specific clinical manifestations (except for episodes of obstructive sleep apnea syndrome) until the age of four years old, when he experienced, for the first time, loss of bladder control followed by recurrent attacks of generalized tonic-clonic seizures in clusters. The video-EEG showed multifocal spike/waves pattern mainly evident in the Fronto-Centro-Parietal regions. The patient started benzodiazepine which managed to control the seizures. Few months later, he presented the first episode of AHC prevailing in right side, which was followed by inability to walk for two months. During this period, the child was fed by nasogastric tube because he was unable to swallow. During the following two years, he was admitted to this Institution for several times in coincidence with the admission of his brother. He manifested frequent episodes of hemiplegia affecting prevalently the right side. The crises were less frequent and less severe compared to those of his brother. He attended the school with almost sufficient progress. At the most recent follow-up visit to the institution (7 $1 / 2$ years old), neurological examination displayed mild ID (IQ 64 WISC III) and notably reduced sensitivity to pain. The paroxysmal episodes of hemiparesis were infrequent (1-2 every three months) and no seizures were reported. The brain MRI, EMG, and NCS were normal. The EEG in the awake state and during sleep

showed the same anomalies as the previous EEG with multifocal spikes and waves (Fig. 2a-b). Treatment received consisted of lorazepam and phenobarbital (Table 1).

\section{Mutation Analysis}

Total genomic DNA was extracted from peripheral blood samples for the mutational analysis, following informed consent by the parents of the families included in the present research.

For twins (Family 1), the molecular diagnosis was carried on ATP1A2 and ATP1A3 genes by sequencing of PCR-amplified DNA. PCR products amplified by LightCycler 480® (Roche Life Science) were determined by direct sequencing on ABI Prism ${ }^{\circledR} 3100$ Genetic Analyzer (Thermo fisher Scientific) according to the manufacturer's protocol. Sequence analysis was carried out by Applied Biosystems DNA Sequencing Analysis v.5.1 and ABI PRISM 310 Data Collection Software Version 3.1 software (ThermoFisher Scientific). Analysis of the pathogenic variant was performed using public repositories of genome variation, such as dbSNP and ClinVar database.

In the family 2, WES analysis using the Illumina TruSight One panel was carried out in both parents and children. The samples were sequenced by using the Illumina NextSeq 500 platform (Illumina Inc.) with 2 $\times 150$ bp paired-end reads. Alignments and variant calls were generated using NextGene software (v2.4.1, 
2015). Variant calls (with coverage $<15 X$ ) were limited to the genes of interest. For the clinical interpretation of genomic variants was used Alamut-Batch (Version 1.4.0, 2015), the high-throughput annotation software for NGS analysis. Alamut Visual (Version 2.7) was used for integrating genetic and predictive information on missense, nonsense, frameshift, and splice-site variants, providing computational algorithms for SIFT, PolyPhen-2 (Version 2.2.2, 2012) and Mutation taster. Variants were annotated for minor allele frequencies in the Exome Aggregation Consortium (ExAC) database (Version $0.3)$, and heterozygous variants with minor allele frequencies $>0.01(1 \%)$ were filtered out. Variants were classified as pathogenic/likely pathogenic/VUS/likely benign/benign according to the 2015 American College of Medical Genetics and Genomics (ACMG) guidelines [9]. The validation of variants classified as pathogenic or likely pathogenic was performed using Sanger sequencing in the probands.

\section{Results}

The candidate gene approach identified in twin girls a pathogenic variant p.Asn773Ser of ATP1A3 gene (rs606231437) associated with AHC-2 (Table 2) [8]. In the siblings, WES analysis found no mutations in ATP1A3 and ATP1A2 genes, identifiying three potentially pathogenic missense variants of GRIN2A, $S C N 1 B$ and $K C N Q 2$ genes in the affected children and their father (Table 2). The missense substitution c.3175T > A (p. Ser1059Thr) in GRIN2A (Glutamate lonotropic Receptor NMDA Type Subunit 2A) gene is the only shared between siblings and the parent and it has not been previously reported in other genomic variation databases. Two missense mutations, c.632G > A (p.Cys211Tyr) in SCN1B (Sodium VoltageGated Channel Beta Subunit 1) gene and c.1870G > A (p.Gly624Arg) in KCNQ2 (Potassium Voltage-Gated Channel Subfamily $Q$ Member 2) gene both found in the firstborn and the father are listed respectively as rs 150721582 and rs771211103 in gnomAD, 1000 Genome project, ClinVar, and dbSNP database. The pathogeniticy prediction done with the MutationTaster tool shows that all of three variants may have a deleterious impact on the function and structure of the protein since the wildtype amino acid position is highly conserved across vertebrate species, and the effects of these changes might also determine potential splice site variants. As shown in the pedigree charting (Fig. 3), the inheritance model of the AHC phenotype in siblings suggests an incomplete or reduced penetrance of gene variants.

\section{Discussion And Conclusions}

The four children, twin girls and two male siblings here reported, are representative example of typical AHC disorder. The presenting clinical manifestations of the children fell within the diagnostic criteria expressed by Krageloh and Aicardi (1980) [10] and Bourgeois et al. (1993) [11]. These diagnostic criteria are: a) onset before the age of 18-months; b) autonomic phenomena; c) cognitive impairment; d) repeated episodes of hemiplegia that sometimes involve both sides of the body; e) evidence of neurological abnormalities such as choreoathetosis; $f$ ) the symptoms disappear during sleeping and resume after waking up. The clinical signs of $\mathrm{AHC}$ are complex, heterogeneous, and follow unique a pattern: their clinical progression tends to occur in sequential distinctive phases, the paroxysmal episodes are often preceded by precipitating factors such as environmental stress, such as bathing and other 
events. Sleeping reliefs the symptoms. Non-paroxysmal features such as DD/ID, epilepsy, motor dysfunction, movement disorders and autonomic manifestations in association with the paroxysmal events are the cornerstone for making the diagnosis of $\mathrm{AHC}$ rather than being considered as comorbidities [12].

Mikati et al. (2000) have demonstrated that the clinical course of patients with AHC evolves in three distinct phases [12]. Phase one begins during the first few months of life and continues for one year and the most common features consist of unilateral nystagmus, ocular deviation, dystonic spells, and developmental delay. Phase two lasts from the age of one to five years, in which the hemiplegic spells become more typical, with a possible frequency of several times each month, and with a duration of several days or even weeks. In this phase, abnormal movements, dystonic attacks, and choreoathetosis are frequently observed. Phase three is represented by fixed neurologic deficits and obvious ID. In this phase, dystonic and hemiplegic episodes become less frequent and less severe. Main clinical manifestations compared to those indicated by Mikati et al. [12] are summarized in Table 1. Identification of this pattern and how the symptoms progress facilitated earlier diagnosis of this disorder bearing in mind that the symptoms are wide particularly regarding the duration and frequency of hemiplegic and dystonic episodes. The beneficial effect of sleep on abnormal paroxysmal features with the disappearance of paroxysmal phenomena and resumption of the normal movements is one of the diagnostic criteria of AHC. This feature is not always reported. However, remission of the paresis may be observed even after a short nap. A study on sleep architecture was carried out in four AHC children and the results showed a normality on the sleep structure, sleep duration, cycle length, rapid eye movement (REM) latency, and REM and slow wave sleep (SWS) percentages [13]. Clinical suspicion starts when the infant presents with abnormal ocular movements such as nystagmus and ocular deviation, head deviation, dystonic spells and unilateral hypotonia, which is usually triggered by several factors including light, sound, exposure to heat or cold, and stress whether physical or psychological. Paroxysmal hemiplegic episodes usually start after the first year of life and usually fluctuate from a side to the other or occur simultaneously on both sides. These episodes may be accompanied by speech impairment, gait incoordination, and movement disorders. The first diagnostic approach is to exclude a diagnosis of epileptic seizures, which can precede, co-occur with, or follow the hemiplegic attacks. A prolonged Video EEG is pivotal for differentiating epileptic seizures from the paroxysmal events of AHC. Routine laboratory examination, plasma amino acids, urine organic acids, blood lactate, pyruvate, urea, ammonia, thyroid functions, arterial blood gases (ABG), MRI and MRI angiography are effective to exclude metabolic disorders and vascular diseases having the same pattern of features such as homocystinuria, organic acidurias (glutaric aciduria), urea cycle disorders (ornithine transcarbamylase deficiency, carbamoyl phosphate synthetase I deficiency, and citrullinemia) and Moyamoya disease. Diagnostic check-up may also include analysis of pterins, 5-methyltetrahydrofolate (5-MTHF) and monoamine metabolites in the cerebrospinal fluid. Regarding the severity of the condition, AHC is usually reported as devastating since hemiplegic features are often associated to other neurologic dysfunctions including DD/ID and epileptic seizures, as discussed in the following paragraphs.

\section{- Cognitive impairment and developmental delay}


Mikati et al. (2000) [12] reported that developmental delay was observed in 40 out of 44 patients enrolled in their study. According to these authors, developmental level correlates with the age of AHC individuals and with the age of onset of the hemiplegic episodes. Although neuropsychological evaluation showed wide variability in functional impairment for cognitive, adaptive and behavioral domains, younger patients demonstrated better results. The authors suggest that remain to establish whether the cognitive delay in AHC individuals is related to the repeated attacks of hemiplegia or a primary effect of the disorder [12]. In the study of Sweney et al. (2009)[14], cognitive impairment was generally defined by families as mild to moderate, and recently a mild cognitive impairment form was reported by Polanowska et al. (2018) [15] in two adult patients, in whom a neuropsychological examination showed a normal or near normal global cognitive functioning, with only some isolated executive functions deficits. In the children here reported the ID was mild and without a progressive course.

\section{- Epileptic seizures}

Epileptic seizures are reported in about $50 \%$ of AHC individuals (Table 3 ) [12, 16-18]. In the study of Mikati et al. (2000) [12] only 8 (19\%) out of 44 patients experienced epileptic seizures, which occurred infrequently in one-half of these patients (three seizures or less). Out of those eight patients, four patients presented with generalised tonic clonic seizures, three with focal clonic seizures, and one with generalised myoclonic seizures. Status epilepticus appeared only in one patient. According to Sweney et al. (2009) [14], 44 (43\%) out $103 \mathrm{AHC}$ individuals showed generalized tonic or tonic clonic seizures. The mean age of onset of epileptic seizures was around 6 years, with 10 (23\%) of the 44 cases who did not experience epileptic episodes until the age of 10 years or later. Ictal seizure EEGs in AHC individuals was reported by Saito et al. (2010) [19]. In another study, status epilepticus appeared in 4 out of 9 patients at the age of 6-16 years [20]. In a report of Uchitel et al. (2019)[21] on 51 patients with AHC, 32 (62.7\%) had focal epilepsy in different cerebral regions, but more frequently frontal region; $11(21.5 \%)$ showed primary generalized seizures tonic clonic, myoclonic, and/or absence. In 8 (15.5\%) patients, epileptic seizures preceded other AHC paroxysmal events. However, according to Heinzen et al. (2012) [5] epileptic seizures may precede the paroxysmal hemiplegic episodes and EEG registration may appear initially normal and then EEG may become epileptiform. In the present cases, the twins up to the age of 19 years never complained of seizures, whereas epileptic seizures were recorded in the siblings presenting with focal seizures with onset in one brother at the age of $3 \frac{1}{2}$ years, and in the other one, at the age of four years. EEG for both siblings showed multifocal spikes and waves expressed mainly in the frontal region. In general, epileptic seizures episodes are reported with low frequency and good response to treatment. A clinical distinction between episodes of hemiplegic attacks and epileptic seizures is not always clear, and the correlation between the epileptic and hemiplegic episodes remains doubtful [19]. Ictal episode was registered in one of the siblings here reported.

\section{- Migraine}

Migraine is a symptom not commonly found in AHC neither in the affected individuals nor in the family history [14]. In the twins here reported the episodes of migraine with aura was one of most consistent 
symptoms.

\section{- $\mathrm{AHC}$ and related disorders}

The cognitive impairment, epileptic seizures, persistent movement disorders, and autonomic dysfunction are considered comorbidities of the AHC disorder. Nevertheless, after refining the symptoms of $\mathrm{AHC}$ by Krägeloh et al.[10] in 1980, then by Bourgeois et al. [11] in 1993, it became obvious that these symptoms may be recorded as primary components of AHC. ATP1A3 has been implicated aside to AHC syndrome to other complex syndromes including the Rapid-onset Dystonia-Parkinsonism [6, 22, 23], and the Cerebellar ataxia, Areflexia, Pes cavus, Optic atrophy, and Sensoryneural hearing loss (CAPOS) syndrome [7, 24, 25]. At their present childhood/adolescent ages no one of the four cases here reported showed clinical features consistent with the uppermentioned syndromes.

\section{- Variability}

Variability in clinical expression of paroxysmal and non-paroxysmal episodes in AHC individuals is well known. In the present cases the intrafamilial variable clinical expression was observed as regard to the course of the intensity and frequency of the hemiplegic episodes and of migraine attacks more pronounced in one of the twins (Table 1). The cognitive impairment was mild in both the twins and no seizures were recorded. In the siblings, the epileptic seizures were more severe in the child who showed more marked hemiplegic attacks. Cognitive impairment was mild in both siblings. It is presumable that epigenetic events have conditioned the intrafamilial clinical variability.

The diagnosis of $\mathrm{AHC}$ is mainly clinical but may be supported by molecular analysis. Typical gene mutations involved in the pathogenesis of AHC are located in ATP1A2 and ATP1A3 genes, but in some cases of AHC, however, recurrent mutations are not found. WES analysis has allowed us to explore the causative genes underlying the pathological mechanisms, which may contribute to the phenotypic variation observed between two siblings. We found in the oldest brother the identical haplotype inherited from the asymptomatic father, constituted of three heterozygous variants in GRIN2A (c.3175T >A), SCN1B (c.632G > A) and KCNQ2 (c.1870G >A) gene, while in the younger child, who has a milder phenotype, only the variant in GRIN2A gene. This mirrors the atypical pattern of inheritance of incomplete or reduced penetrance of AHC-2 mutations. The GRIN2A gene encoding the NMDA receptor (NMDAR) subunit GluN2A has been suggested to constitute a locus for mutations in a subset of individuals with early-onset seizures [26]. A literature overview shows that pathogenic or likely pathogenic variants are spread over nearly the entire gene, but the majority of null variants in healthy individuals are observed in the exon 14, which encodes nearly the complete C-terminal domain. These variants do not exhibit effects because this region represents the protein region tolerant to genetic variation in the general population. No missense variants in the intracellular C-terminal domain of GluN2A (beyond amino acid position 838) have been found to fulfil ACMG criteria for being pathogenic or likely pathogenic [26]. According to the pathogenicity prediction, the GRIN2A (c.3175T > A; p.Ser1059Thr) variant is likely pathogenic leading to significant alterations of the protein properties and splice site changes. It can be assumed as a first delineation of a phenotypically causal variant observed in the C-terminal domain of the protein 
associated with a reduced penetrance, since normal individuals are more likely to pass on their pathogenic variants to the offspring [26]. Additional two likely pathogenic variants implicated in childhood epilepsies [27-29] one of SCN1B (rs150721582) gene and another one of KCNQ2 (rs771211103) gene, were identified in the firstborn, who carried the more severe phenotype compared to the younger brother possibly due to harmful and cumulative effects driven by these mutations together with the GRIN2A variant. Some variant forms of AHC, AHC-related disorders and channelopathies are characterized by mutations involving glutamate, sodium and potassium channels [30-32]. The identification of mutations in GRIN2A, SCN1B and KCNQ2 genes shows the power of some low penetrant variants in eliciting the phenotypic spectrum of $A H C$ not associated with the primary genetic risk factors in ATP1A2 and ATP1A3 genes [28, 32]. Analyzing the role of these genes both individually and in association with each other, we cannot exclude to be likely eligible as risk factors for AHC since the number and type of alteration in the expression of encoded proteins may disrupt some functional connections in the transcriptional regulatory network leading to the clinical variability of the disease.

To date, no drugs are available to cure AHC. The treatment usually comprises multiple drug therapy regimen. The aim of these therapeutic agents is prophylactic against the paroxysmal attacks. Flunarizine is a calcium channel blocker that has been widely indicated as the most effective drug for AHC treatment. This drug was first proposed by Casaer and Azou (1984)[33] in one child, and then in 12 children with AHC [34]. Subsequently to the first indication [33], the drug was tried by Sakuragawa (1992) on 23 patients [3] and by Burgeois and Aicardi (1993) [35] on 17 patients. The results obtained by these authors suggested that flunarizine therapy reduced the duration and severity of hemiplegic attacks, but did not interfere with the natural course of the disease. To note that no severe side-effects have been seen in any patients during the time of treatment [20]. In the study of Mikati et al. (2000) [12], 27 out of 44 patients affected by AHC showed a good response to Flunarizine. According to these authors [12], a clinically worthy reduction in the frequency and/or severity was found in 21 patients $(78 \%)$. Flunarizine eliminated the hemiplegic attacks completely in one patient (4\%), improved the attacks partially in two patients (7\%), and was without any effect in four patients (15\%). Sweney et al. (2009)[14] reported improvement in dystonic or hemiplegic episodes in 48 out of 80 patients using flunarizine and 21 out of 55 patients using benzodiazepines. In an Italian study conducted on 30 patients with AHC, Pisciotta et al. (2016)[6] reported that flunarizine resulted in reduction in the duration and frequency of hemiplegic attacks in $50 \%$ of the patients and decreased their intensity in $32.1 \%$, the decreased intensity was more evident in the younger patients. No correlation between developmental outcome and duration of treatment was reported by these authors [6]. Flunarizine seems to be the best treatment option for the episodic hemiplegic attacks in patients with AHC. In our patients the treatment with flunarizine was conducted irregularly by the parents, however it was able to reduce frequency and intensity of hemiplegic attacks when regularly used. Recently, new drugs and new treatment modalities have been proposed. Triheptanoin is a triglyceride composed of three seven-carbon (C7:0) fatty acids. It acts by providing anaplerotic substrates for Krebs cycle and appears to increase the efficacy of the ketogenic diet. It was tested on 10 patients with AHC [20]. The trial with this drug was unsuccessful and failed to reduce the paroxysmal events. Aripripazole, an atypical antipsychotic drug was proposed by Dundar et al. (2019)[36] in a 12-year-old male with AHC 
who was not responding to any type of classical treatment; Aripripazole showed a reduction in the duration and frequency of hemiplegic episodes. Verapamil is a calcium-channel blocker and has the same mechanism of action of flunarizine. It was administered in a 5-year-old boy with AHC presenting with poorly controlled epileptic seizures and developmental delay [37]. Notable reduction in frequency, severity, and duration of the hemiplegic attacks was obtained with the use of this drug [38]. In association with flunarizine, anticonvulsant has been applied in the treatment of the epileptic attacks using benzodiazepine, carbamazepine, barbiturates and valproate [39]. In the siblings, lorazepam and benzodiazepines managed to control the seizures.

$\mathrm{AHC}$ is a complex, often serious disorder in which the hemiplegic episode is only one sign even if the most remarkable, of several other body-system impairments involving the autonomic nervous system, musculoskeletal system, and the brain, with epileptic seizures and cognitive dysfunction as a relevant clinical association. The narrow association and correlation of these clinical signs leads us to conclude that clinical involvement of AHC must be extended and included in the term "AHC spectrum disorder".

\section{Abbreviations}




\begin{tabular}{|ll|}
\hline ACMG & $\begin{array}{l}\text { American College of Medical Genetics } \\
\text { and Genomics }\end{array}$ \\
AHC & Alternating Hemiplegia of Childhood \\
ATterial Blood Gases & ABS \\
ATP2 & $\begin{array}{l}\text { ATPase Na+/K+ Transporting Subunit } \\
\text { Alpha 2 }\end{array}$ \\
\hline ATP1A3 & ATPase Na+/K+ Transporting Subunit \\
Cerebellar ataxia-areflexia-pes cavus-optic atrophy- & Alpha 3 \\
sensorineural hearing loss & CAPOS \\
Developmental Delay & \\
Electroencephalogram & DD \\
GRIN2A & EEG \\
Intellectual Disability & Glutamate lonotropic Receptor NMDA \\
KCNQ2 & Type Subunit 2A \\
Magnetic Resonance Imaging & ID \\
NMDA receptor & Potassium Voltage-Gated Channel \\
Obstructive Sleep Apnea Syndrome & Subfamily Q Member 2 \\
Rapid Eye Movement & MRI \\
SCN1B & NMDAR \\
Slow Wave Sleep & OSAS \\
Whole Exome Sequencing & REM \\
& Sodium Voltage-Gated Channel Beta \\
& Subunit 1 \\
& SWS \\
\hline
\end{tabular}

\section{Declarations}

\section{Ethics approval and consent to participate}

The research was conducted ethically in accordance with the World Medical Association Declaration of Helsinki and approved by ethics committee of the University of Catania, Italy (Ethical Committee Catania 1 Clinical Registration n. 95/2018/PO). Written informed consent was obtained from parents.

\section{Consent for publication}


Written informed consent for publication of their clinical details and/or clinical images was obtained from the patient/parent/guardian/relative of the patient. A copy of the consent form is available for review by the Editor of this journal.

\section{Availability of data and materials}

All data generated or analysed during this study are included in this published article.

\section{Competing interests}

The authors have no conflicts of interest to declare.

\section{Funding}

This research did not receive any specific grant from funding agencies in the public, commercial, or notfor-profit sectors.

\section{Authors' Contributions}

P.P. worked with and helped gather patient data, drafted and redrafted the present manuscript. X.G.P., S.Y.C. and D.K.J. helped analyze the genetic data. X.G.P interpret the literature relevant to the mutation and realize the figures. G.I. followed the twins since their first referral to our institutions in Italy, reviewed the clinical history and revised the manuscript. N.M., S.D.M. and R.F. contributed to the clinical understanding of the case and revised the manuscript. G.C., E.P. and M.R. were called as consultants regarding the diagnosis of alternating hemiplegia in childhood and reviewed the manuscript. All authors read and approved the final manuscript.

\section{Acknowledgements}

We thank parents of both families for availability of publishing medical history of their children.

\section{References}

1. Verret S, Steele JC. Alternating hemiplegia in childhood: a report of eight patients with complicated migraine beginning in infancy. Pediatrics. 1971 Apr;47(4):675-80. PubMed PMID: 5089756.

2. Aicardi J, Bourgeois, M., Goutieres, F. . Alternating hemiplegia of childhood: clinical findings and diagnostic criteria. . Andermann F, Aicardi J, Vigevano F, editors Alternating Hemiplegia of Childhood New York: Raven Press. 1995:p 3-18. 
3. Sakuragawa N. Alternating hemiplegia in childhood: 23 cases in Japan. Brain \& development. 1992 Sep;14(5):283-8. PubMed PMID: 1456380.

4. Bassi MT, Bresolin N, Tonelli A, Nazos K, Crippa F, Baschirotto C, et al. A novel mutation in the ATP1A2 gene causes alternating hemiplegia of childhood. Journal of medical genetics. 2004 Aug;41(8):621-8. PubMed PMID: 15286158. Pubmed Central PMCID: 1735877.

5. Heinzen EL, Swoboda KJ, Hitomi Y, Gurrieri F, Nicole S, de Vries B, et al. De novo mutations in ATP1A3 cause alternating hemiplegia of childhood. Nature genetics. 2012 Sep;44(9):1030-4. PubMed PMID: 22842232. Pubmed Central PMCID: 3442240.

6. Pisciotta L, Gherzi M, Stagnaro M, Calevo MG, Giannotta M, Vavassori MR, et al. Alternating Hemiplegia of Childhood: Pharmacological treatment of 30 Italian patients. Brain \& development. 2017 Jun;39(6):521-8. PubMed PMID: 28249736.

7. Sweney MT, Newcomb TM, Swoboda KJ. The expanding spectrum of neurological phenotypes in children with ATP1A3 mutations, Alternating Hemiplegia of Childhood, Rapid-onset DystoniaParkinsonism, CAPOS and beyond. Pediatric neurology. 2015 Jan;52(1):56-64. PubMed PMID: 25447930. Pubmed Central PMCID: 4352574.

8. Pavone P, Pappalardo XG, Incorpora G, Falsaperla R, Marino SD, Corsello G, et al. Long-term follow-up and novel genotype-phenotype analysis of monozygotic twins with ATP1A3 mutation in Alternating Hemiplegia of Childhood-2. European journal of medical genetics. 2020 Aug;63(8):103957. PubMed PMID: 32454213.

9. Richards S, Aziz N, Bale S, Bick D, Das S, Gastier-Foster J, et al. Standards and guidelines for the interpretation of sequence variants: a joint consensus recommendation of the American College of Medical Genetics and Genomics and the Association for Molecular Pathology. Genetics in medicine : official journal of the American College of Medical Genetics. 2015 May;17(5):405-24. PubMed PMID: 25741868. Pubmed Central PMCID: 4544753.

10. Krageloh I, Aicardi J. Alternating hemiplegia in infants: report of five cases. Developmental medicine and child neurology. 1980 Dec;22(6):784-91. PubMed PMID: 7450304.

11. Bourgeois M, Aicardi J, Goutieres F. Alternating hemiplegia of childhood. The Journal of pediatrics. 1993 May;122(5 Pt 1):673-9. PubMed PMID: 8496742.

12. Mikati MA, Kramer U, Zupanc ML, Shanahan RJ. Alternating hemiplegia of childhood: clinical manifestations and long-term outcome. Pediatric neurology. 2000 Aug;23(2):134-41. PubMed PMID: 11020638.

13. Ricci S. Sleep studies of children with alternating hemiplegia of childhood. . Alternating Hemiplegia of Childhood Andermann, Aicardi, and Vigevano. 1995:95-8.

14. Sweney MT, Silver K, Gerard-Blanluet M, Pedespan JM, Renault F, Arzimanoglou A, et al. Alternating hemiplegia of childhood: early characteristics and evolution of a neurodevelopmental syndrome. Pediatrics. 2009 Mar;123(3):e534-41. PubMed PMID: 19254988.

15. Polanowska KE, Dziezyc K, Rosewich H, Ohlenbusch A, Seniow JB. Alternating Hemiplegia of Childhood in Two Adult Patients with a Mild Syndrome. Cognitive and behavioral neurology : official 
journal of the Society for Behavioral and Cognitive Neurology. 2018 Dec;31(4):214-9. PubMed PMID: 30562231.

16. Panagiotakaki E, De Grandis E, Stagnaro M, Heinzen EL, Fons C, Sisodiya S, et al. Clinical profile of patients with ATP1A3 mutations in Alternating Hemiplegia of Childhood-a study of 155 patients. Orphanet journal of rare diseases. 2015 Sep 26;10:123. PubMed PMID: 26410222. Pubmed Central PMCID: 4583741.

17. Kansagra S, Mikati MA, Vigevano F. Alternating hemiplegia of childhood. Handbook of clinical neurology. 2013;112:821-6. PubMed PMID: 23622289.

18. Sasaki M, Ishii A, Saito Y, Morisada N, lijima K, Takada S, et al. Genotype-phenotype correlations in alternating hemiplegia of childhood. Neurology. 2014 Feb 11;82(6):482-90. PubMed PMID: 24431296.

19. Saito $Y$, Inui T, Sakakibara T, Sugai K, Sakuma H, Sasaki M. Evolution of hemiplegic attacks and epileptic seizures in alternating hemiplegia of childhood. Epilepsy research. 2010 Aug;90(3):248-58. PubMed PMID: 20580529.

20. Rosewich H, Ohlenbusch A, Huppke P, Schlotawa L, Baethmann M, Carrilho I, et al. The expanding clinical and genetic spectrum of ATP1A3-related disorders. Neurology. 2014 Mar 18;82(11):945-55. PubMed PMID: 24523486.

21. Uchitel J, Helseth A, Prange L, McLean M, Ghusayni R, Sachdev M, et al. The epileptology of alternating hemiplegia of childhood. Neurology. 2019 Sep 24;93(13):e1248-e59. PubMed PMID: 31484714.

22. Dobyns WB, Ozelius LJ, Kramer PL, Brashear A, Farlow MR, Perry TR, et al. Rapid-onset dystoniaparkinsonism. Neurology. 1993 Dec;43(12):2596-602. PubMed PMID: 8255463.

23. Brashear A, Dobyns WB, de Carvalho Aguiar P, Borg M, Frijns CJ, Gollamudi S, et al. The phenotypic spectrum of rapid-onset dystonia-parkinsonism (RDP) and mutations in the ATP1A3 gene. Brain : a journal of neurology. 2007 Mar;130(Pt 3):828-35. PubMed PMID: 17282997.

24. Nicolaides P, Appleton RE, Fryer A. Cerebellar ataxia, areflexia, pes cavus, optic atrophy, and sensorineural hearing loss (CAPOS): a new syndrome. Journal of medical genetics. 1996 May;33(5):419-21. PubMed PMID: 8733056. Pubmed Central PMCID: 1050615.

25. Burnashev N, Szepetowski P. NMDA receptor subunit mutations in neurodevelopmental disorders. Current opinion in pharmacology. 2015 Feb;20:73-82. PubMed PMID: 25498981.

26. Strehlow V, Heyne HO, Vlaskamp DRM, Marwick KFM, Rudolf G, de Bellescize J, et al. GRIN2A-related disorders: genotype and functional consequence predict phenotype. Brain : a journal of neurology. 2019 Jan 1;142(1):80-92. PubMed PMID: 30544257. Pubmed Central PMCID: 6308310.

27. Orrico A, Galli L, Grosso S, Buoni S, Pianigiani R, Balestri P, et al. Mutational analysis of the SCN1A, SCN1B and GABRG2 genes in 150 Italian patients with idiopathic childhood epilepsies. Clinical genetics. 2009 Jun;75(6):579-81. PubMed PMID: 19522081.

28. Symonds JD, Zuberi SM. Genetics update: Monogenetics, polygene disorders and the quest for modifying genes. Neuropharmacology. 2018 Apr;132:3-19. PubMed PMID: 29037745. 
29. Maljevic S, Reid CA, Petrou S. Models for discovery of targeted therapy in genetic epileptic encephalopathies. Journal of neurochemistry. 2017 Oct;143(1):30-48. PubMed PMID: 28742937.

30. Rho JM, Chugani HT. Alternating hemiplegia of childhood: insights into its pathophysiology. Journal of child neurology. 1998 Jan;13(1):39-45. PubMed PMID: 9477247.

31. Auvin S, Joriot-Chekaf S, Cuvellier JC, Vallee L. 'Familial alternating hemiplegia of childhood or channelopathy? A report with valuable pathophysiological implications'. Developmental medicine and child neurology. 2004 Jul;46(7):500; author reply 1. PubMed PMID: 15230465.

32. Olson HE, Poduri A, Pearl PL. Genetic forms of epilepsies and other paroxysmal disorders. Seminars in neurology. 2014 Jul;34(3):266-79. PubMed PMID: 25192505. Pubmed Central PMCID: 4834292.

33. Casaer P, Azou M. Flunarizine in alternating hemiplegia in childhood. Lancet. 1984 Sep 8;2(8402):579. PubMed PMID: 6147627.

34. Casaer P. Flunarizine in alternating hemiplegia in childhood. An international study in 12 children. Neuropediatrics. 1987 Nov;18(4):191-5. PubMed PMID: 3320807.

35. Bourgois MA, S. . The treatment of Alternating hemiplegia of childhood:treatment with flunarizine. . Alternating hemiplegia of childhood Eds Andermann F, Aicardi J \& Vigevano F New York: Raven Press. 1994:191-3. .

36. Dundar NO, Cavusoglu D, Kaplan YC, Hasturk MO. An Option to Consider for Alternating Hemiplegia of Childhood: Aripiprazole. Clinical neuropharmacology. 2019 May/Jun;42(3):88-90. PubMed PMID: 30893129.

37. Van Hillegondsberg LS, Michaelis IA. Alternating hemiplegia of childhood: First South African case report and verapamil as a possible treatment option. South African medical journal $=$ SuidAfrikaanse tydskrif vir geneeskunde. 2019 Feb 26;109(3):152-3. PubMed PMID: 30834869.

38. Brashear A, Sweadner KJ, Cook JF, Swoboda KJ, Ozelius L. ATP1A3-Related Neurologic Disorders. In: Adam MP, Ardinger HH, Pagon RA, Wallace SE, Bean LJH, Stephens K, et al., editors. GeneReviews((R)). Seattle (WA)1993.

39. Neville BG, Ninan M. The treatment and management of alternating hemiplegia of childhood. Developmental medicine and child neurology. 2007 Oct;49(10):777-80. PubMed PMID: 17880649.

\section{Tables}

Due to technical limitations, tables 1-3 are only available as a download in the Supplemental Files section.

\section{Figures}




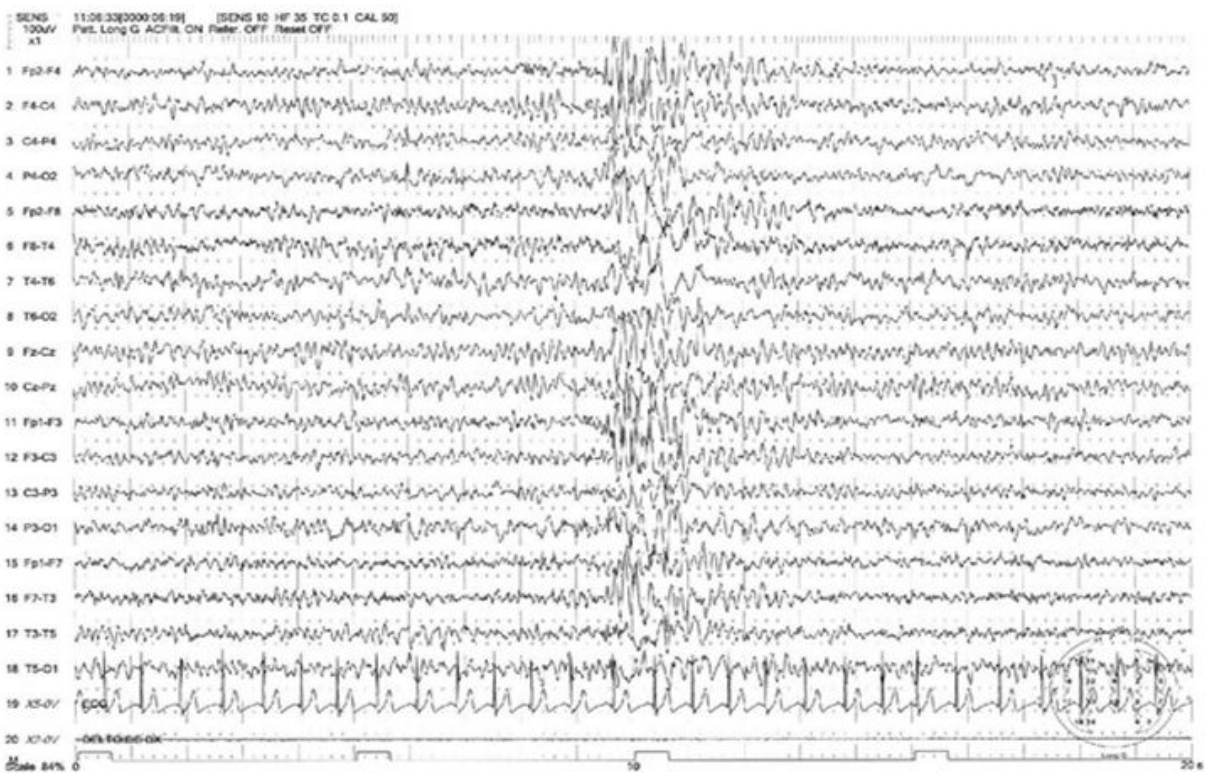

Fig 1a

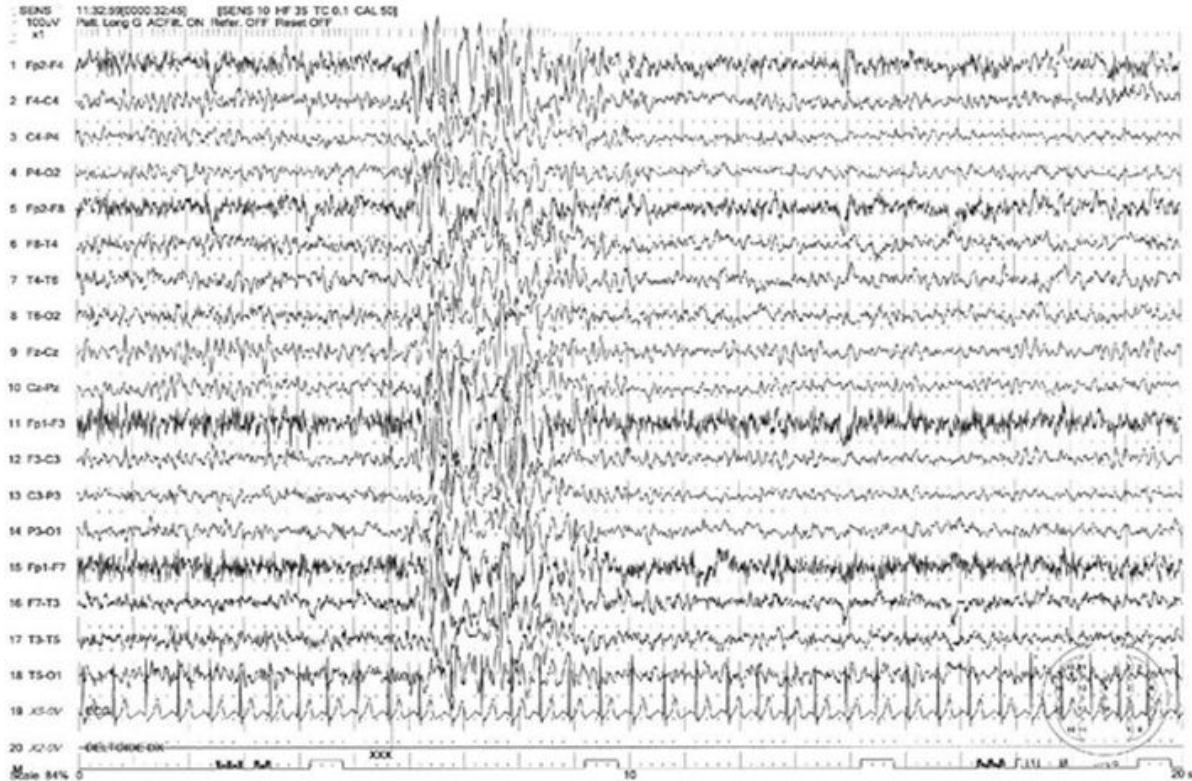

Fig $1 b$

Figure 1

1a- Family 2. Brother 1. EEG at 8 years old showing multifocal spike/waves prevalently in the frontal regions. $1 \mathrm{~b}$ - Family 2. Brother 1. Ictal EEG showing multifocal spikes and waves. 


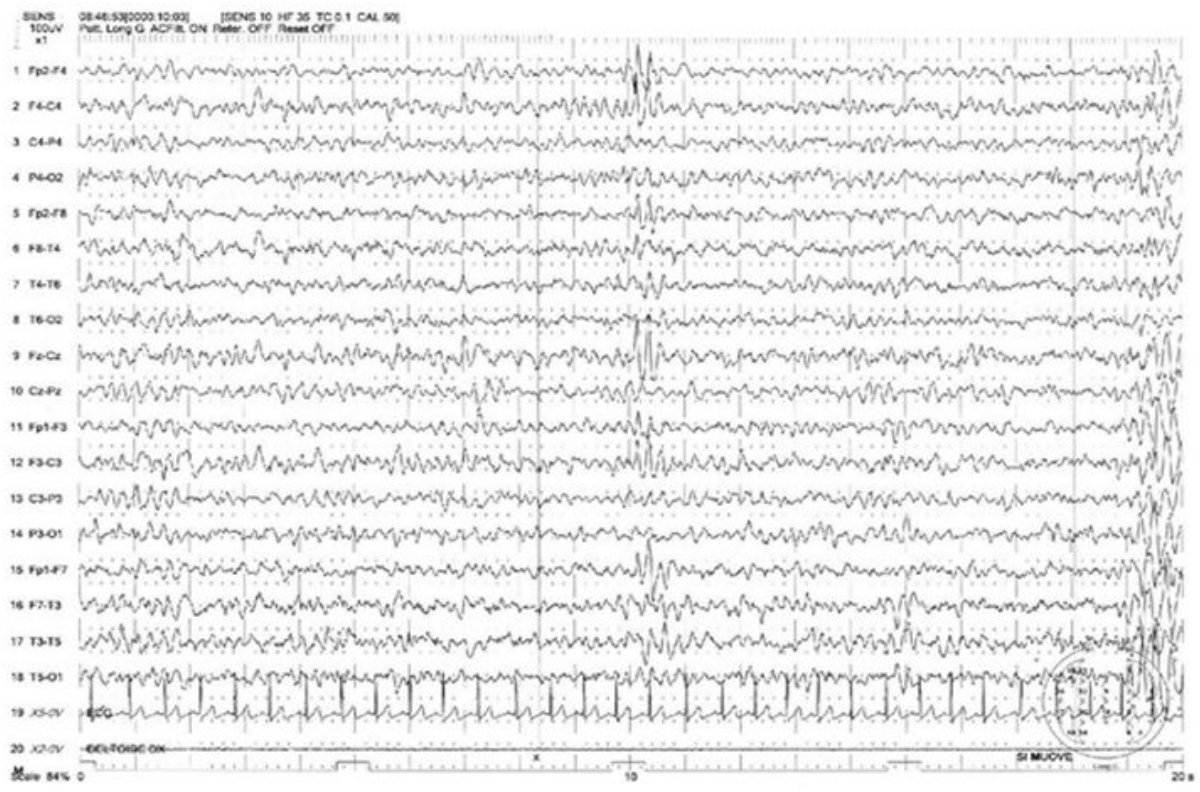

Fig $2 a$

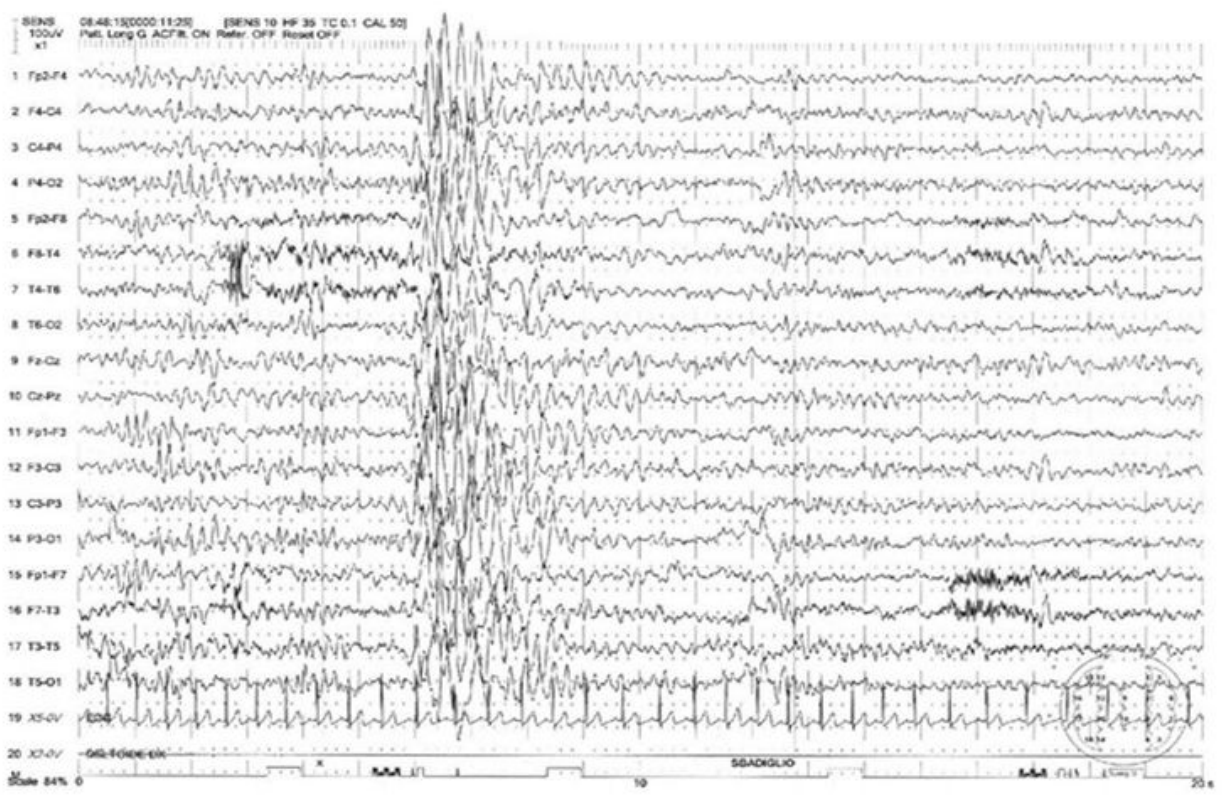

Fig $2 b$

Figure 2

2a - Family 2. Brother 2. EEG at 4 years old showing multifocal spike/waves prevalently in fronto-centroparietal regions. $2 \mathrm{~b}-$ Family 2. Brother 2. EEG at 4 years old showing multifocal spike/waves with generalization. 


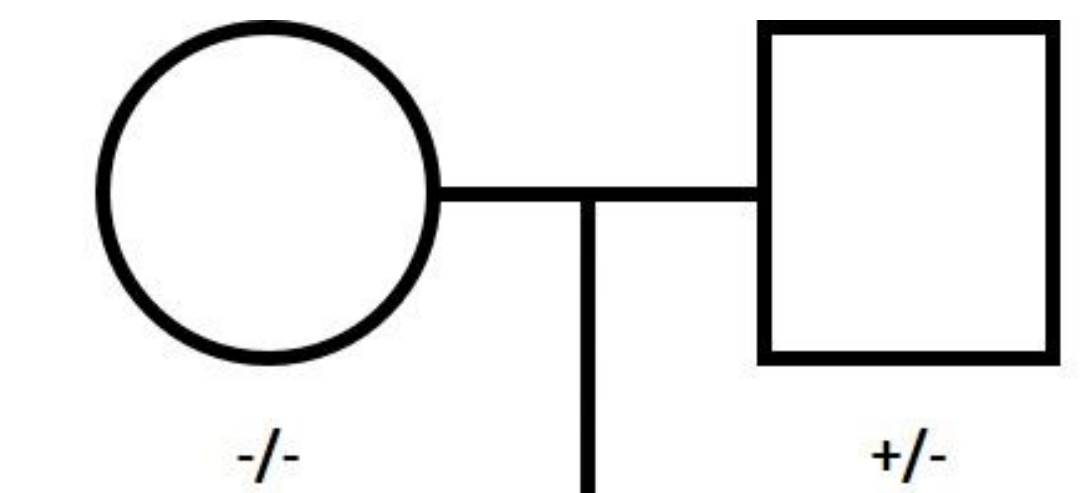

GRIN2A c.3175T>A ; p.Ser1059Thr

SCN1B c.632G>A; p.Cys211Tyr

KCNQ2 c.1870G>A; p.Gly624Arg
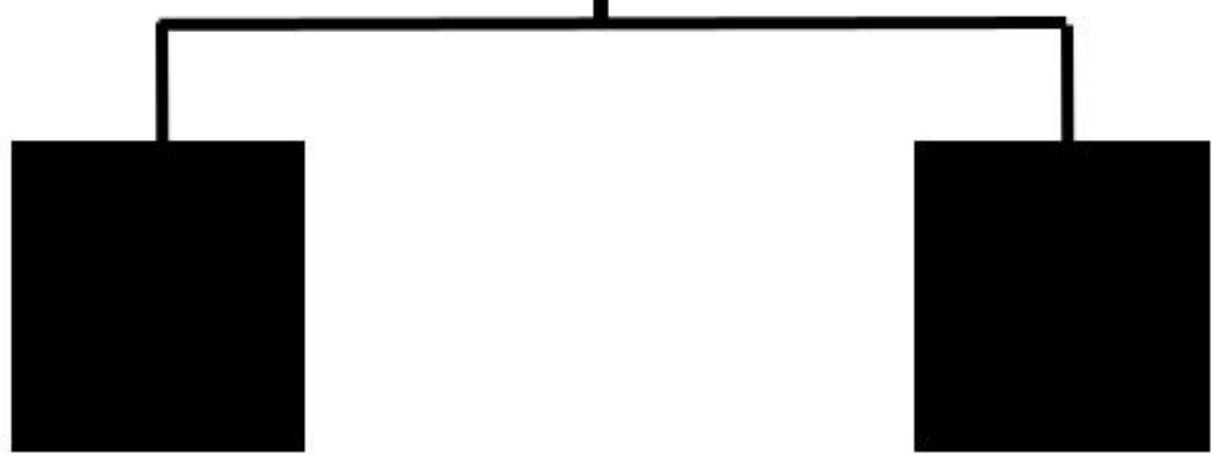

$+/-$

$+/-$

GRIN2A c.3175T>A ; p.Ser1059Thr

GRIN2A c.3175T>A ; p.Ser1059Thr

SCN1B c.632G>A; p.Cys211Tyr

KCNQ2 c.1870G>A; p.Gly624Arg

\section{Figure 3}

Family 2 pedigree and heterozygous likely pathogenic variants identified in GRIN2A, SCN1B and KCNQ2 genes. Affected probands (siblings) are indicated by arrow. Variants detected in siblings are inherited from the father who has a normal phenotype revealing an incomplete penetrance of AHC-2.

\section{Supplementary Files}

This is a list of supplementary files associated with this preprint. Click to download. 
- Tables13.xlsx

Page 21/21 